RESEARCH ARTICLE

\title{
Evaluation of Trypanocidal and Antioxidant Activities of a Selected Series of 3-amidocoumarins
}

\author{
Mauricio Moncada-Basualto ${ }^{\mathrm{a}, \mathrm{b}}$, Michel Lapier ${ }^{\mathrm{c}}$, Juan Diego Maya ${ }^{\mathrm{c}}$, Betty Matsuhiro ${ }^{\mathrm{b}}$, Claudio Olea- \\ Azar $^{\mathrm{a},{ }^{*}}$, Giovanna L. Delogu ${ }^{\mathrm{d}}$, Eugenio Uriarte ${ }^{\mathrm{e}, \mathrm{f}}$, Lourdes Santana ${ }^{\mathrm{e}}$ and Maria João Matos ${ }^{\mathrm{e}, * *}$
}

${ }^{a}$ Laboratory of Free Radicals and Antioxidants, Faculty of Chemical and Pharmaceutical Sciences, University of Chile, Sergio Livingstone Polhammer 1007, Independencia, Santiago, Chile; ${ }^{b}$ Laboratory of Carbohydrates, Faculty of Chemistry and Biology, University of Santiago of Chile, Libertador Bernardo O’Higgins 3363, Estación Central, Santiago, Chile; 'Department of Molecular and Clinical Pharmacology, Faculty of Medicine, University of Chile, Santiago, Chile; ${ }^{d}$ Department of Life Sciences and Environment - Section of Pharmaceutical Sciences, University of Cagliari $09124 \mathrm{Ca}-$ gliari, Italy; ${ }^{e}$ Department of Organic Chemistry, Faculty of Pharmacy, University of Santiago de Compostela, 15782 Santiago de Compostela, Spain; Instituto de Ciencias Químicas Aplicadas, Universidad Autónoma de Chile, 7500912 Santiago, Chile

A R T I C LE H I S T O R Y

Received: July 26, 2017

Revised: April 12, 2018

Accepted: April 12, 2018

DOI:

$10.2174 / 1573406414666180419113437$

\begin{abstract}
Background: Neglected diseases are becoming more prevalent due to globalization. This has inspired active research in the development of new drugs for the treatment of parasitic diseases such as Chagas disease.
\end{abstract}

Objectives: With the aim of finding new trypanocidal agents, we report the in vitro evaluation of a new series of 3-amidocoumarins with or without hydroxyl substituents at position 4 of the coumarin ring.

Methods: Electrochemical and biological assays were performed in order to assess the antioxidant and trypanocidal potential of these compounds and to better understand the mechanisms involved in their activity.

Results: Most of the studied compounds showed high trypanocidal activity against both epimastigote and trypomastigote forms, with $\mathrm{IC}_{50}$ values in the low micromolar range. Some of them have greater activity and selectivity than the reference compound, nifurtimox.

Conclusion: Compound $\mathbf{2}$ is the most active of this series, being also non-cytotoxic against murine RAW 264.7 macrophages. Electrochemical and radical scavenging experiments were carried out, providing new information about the profile of the best derivatives, and the potential therapeutic application of the new 3-amidocoumarins.

Keywords: 3-Amidocoumarins, trypanosoma cruzi, trypanocidal, antioxidant, chagas disease, protozoan parasite.

\section{INTRODUCTION}

Chagas disease is a pathology caused by the protozoan parasite Trypanosoma cruzi (T. cruzi) that affects approximately 7 million people worldwide and causes more than 7000 deaths per year, according to statistics of PAHO (Pan American Health Organization) and WHO (World Health Organization) [1]. Although endemic in Latin America, the disease has also been spread to North America, Europe and several Western Pacific countries. Outside the endemic regions, the highest burden occurs in the USA, where 300,000 people live with the disease [2].

\footnotetext{
*Address correspondence to this author at the Department of Organic Chemistry, Faculty of Pharmacy, University of Santiago de Compostela, 15782 Santiago de Compostela, Spain; Tel: +34981528070 , E-mails: mariacmatos@gmail.com; and Laboratory of Free Radicals and Antioxidants, Faculty of Chemical and Pharmaceutical Sciences, University of Chile, Sergio Livingstone Polhammer 1007, Independencia, Santiago-Chile; Email:colea@uchile.cl
}

The only two available drugs used for the acute phase of Chagas disease, avoiding that patients come to a chronic period (nifurtimox -NFX- and benznidazole), have several drawbacks such as poor efficacy, toxicity, resistance and multiple side effects. Therefore, there is an urgent need for new, more effective and safer drugs for human use [3].

During infection and development of Chagas disease, reactive species of oxygen (ROS) can be produced due to tissue destruction by parasite toxic secretions, immunologically mediated cytotoxic reactions and secondary myocardial damage [4-6]. These species are responsible for the oxidative damage, causing lipid peroxidation, protein modification and DNA damage [7]. It was described that antioxidant compounds, such as flavonoids (coumarin isomers), stimulate the host immune system, allowing the reduction of protozoan parasites [8]. In addition, it was found that coumarins, besides their important role as antioxidants, can display several biological activities, such as anticoagulant, anti- 
inflam-matory, spasmolytic and antiparasitic. Therefore, these compounds can be explored as an alternative for treating Chagas disease [9-13].

\section{RESULTS AND DISCUSSION}

Our research group synthesized a series of 4hydroxycoumarins, and performed both antioxidant and trypanocidal studies, finding that these compounds display significant activity against the epimastigote form [14]. In addition, we have synthesized a series of 3-amidocoumarins with interesting profiles both as antioxidants and trypanocidals [4].

In order to obtain information about the trypanocidal mechanism of action of a series of 3-carboxamidocoumarins, we studied the parasite mitochondrial membrane potential [15]. The generation of oxidative stress in the parasite can be responsible for the mitochondrial dysfunction, essential for the survival of parasite and the regulation of processes related to energy balance and modulation of apoptosis [16]. Moreover, it was reported that coumarins can inhibit glyceraldehyde-3-phosphate dehydrogenase (GAPDH), which is important in the trypanosomatid glycolytic pathway and may be an intracellular target [3].

Based on this preliminary data, a series of 3-amidocoumarins (Scheme 1) was synthesized. Influence of a hydroxyl group at position 4 of the coumarin scaffold was evaluated.

The described derivatives were efficiently synthesized according to the protocol outlined in Scheme 1. Coumarins 1-9 were prepared starting from 3-aminocoumarin or from 4-hydroxy-3-aminocoumarin, which were synthesized as previously described [17-22].

The 3-amino-4-hydroxycoumarin was prepared by a reduction of the commercially available 4-hydroxy-3nitrocoumarin, in ethanol, using $\mathrm{Pd} / \mathrm{C}$ as catalyst, in $\mathrm{H}_{2}$ atmosphere for $5 \mathrm{~h}$, with a yield of $90 \%$ (purified by flash chromatography, using n-hexane/ethyl acetate 9:1 as solvents). An acylation reaction of the 3-aminocoumarins, in the presence of the conveniently substituted acid chloride, using pyridine in dichloromethane, from $0{ }^{\circ} \mathrm{C}$ to room temperature, overnight, afforded the desired 3-amidocoumarins (1-9) in yields between $80 \%$ and $90 \%$ (purified by flash chromatography, using n-hexane/ethyl acetate 9:1 as solvents).

Oxidation potential was determined by using differential pulse voltammetry (DPV). The oxidation potential is a physicochemical parameter closely related to the antioxidant capacity. All studied compounds showed a single oxidation peak. The hydroxylated derivatives proved to be those having lower oxidation potential (Table 1). It was found that the introduction of an acrylamide at position 3 of the coumarin(compound 2), shifted the potential to more positive values, preventing oxidation of the molecule, comparing to the acetamide group (compound 1). Also, the introduction of five-membered heterocyclics (furan and thiophene) in the carboxamide, slightly decreased the oxidation potentials comparing to the coumarins containing an acrylamide group (Fig. 1).

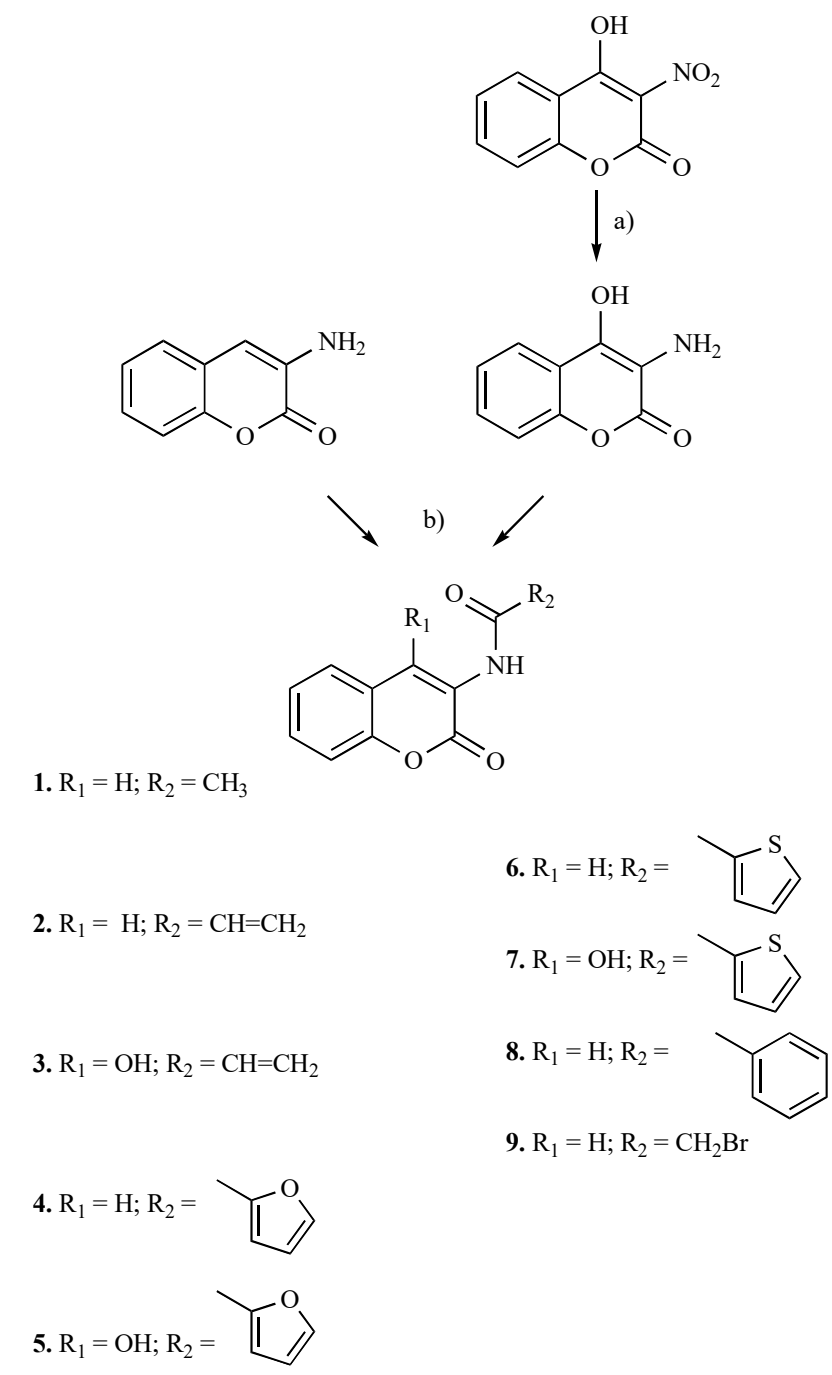

Scheme 1. Chemical structures of the studied compounds. Reagents and conditions: a) hydrogen gas $\left(\mathrm{H}_{2}\right)$, Ethanol as solvent, Palladium on carbon $(\mathrm{Pd} / \mathrm{C})$ used as a catalyst, stirring at room temperature for $5 \mathrm{~h}$; b) Substituted acid chloride $\left(\mathrm{R}_{2} \mathrm{COCl}\right)$, pyridine, dichloromethane, $0{ }^{\circ} \mathrm{C}$ to room temperature, overnight. All the details are described in references [17-22].

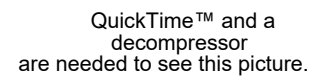

Fig. (1). DPV of compounds 2-5, in DMF, at $20 \mathrm{mV} \mathrm{s}^{-1}$. 


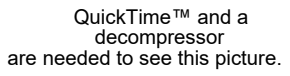

Fig. (2). A) Cyclic voltammograms for $1 \mathrm{mmol} / \mathrm{L}$ of compound 8, v $=0.05-2.5 \mathrm{~V} / \mathrm{s}$. B) Ratio of Epa (V) versus log scan speed (v). C) Ratio of $\log$ Ipa (V) versus log scan speed (v).

Based on the oxidation potentials (Table 1), the antioxidant capacity of the compounds can be ranked as: $\mathbf{1}>\mathbf{7} \approx \mathbf{3} \approx$ $\mathbf{5}>\mathbf{6}>\mathbf{8}>\mathbf{4}>\mathbf{2} \approx \mathbf{9}$. The hydroxylated derivatives presented similar oxidation potentials, higher than the standard antioxidant Trolox (water-soluble $\alpha$-tocopherol derivative).

The electrochemical behaviour of the 3-amidocoumarins was also studied by cyclic voltammetry (CV). The nonhydroxylated compounds $(\mathbf{1}, \mathbf{2}, \mathbf{4}, \mathbf{6}, \mathbf{8}$ and 9) showed similar anodic behaviour. A single anodic peak without a cathodic signal was found for all derivatives. This corresponds to an irreversible oxidation, under the studied conditions (Fig. 2A). In addition, the anodic peak potential (Epa) was dependent on the sweep speed, supporting the evidence of irreversibility of the oxidation of these molecules (Fig. 2B) [23].

To determine if the oxidation process was controlled by an adsorption or diffusion phenomenon, the logarithm of the anodic peak (log (Ipa)) was plotted against the logarithm of the process speed $(\log (\mathrm{v}))$ (Fig. 2C). The value of the slope indicates the process involved. According to Rodriguez et al, for a controlled diffusion process, the value is close to 0.5 , and for a controlled adsorption process, the value is close to 1.0 [24]. The slope values for the non-hydroxylated compounds indicate an adsorptive diffusion controlled process without the interference of the working electrode [25].

For the hydroxylated 3-amidocoumarins $(\mathbf{3}, 5$ and 7$)$, the voltammograms indicate an irreversible oxidation, determined by the criteria of the dependence of the anodic peak with the process speed (Fig. 3).
The oxidation process of both hydroxylated and nonhydroxylated coumarins occurred by diffusion into the glassy carbon electrode, as determined by the described criteria.

Comparing with the previously studied 4-hydroxycoumarins whose oxidation potential was $1.33 \mathrm{~V}$ [26], it can be established a different electro-oxidation mechanism for the currently studied molecules. The introduction of electron withdrawing groups hampered the oxidation of this type of systems, as described by us in a previous paper [4].

To deeply study the mechanism of oxidation of 3amidocoumarins, the electrolytic generation of radicals, obtained by DPV, was performed. Detection and characterization of these radical species were carried out by the electronic spin resonance (ESR), using a spin trapping [27]. The electrochemical oxidation of the substituted 3-amidocoumarins was performed under the same conditions as for the CV but using $N$-tert-butyl- $\alpha$-phenylnitrone (PBN) as a spin trapping. This trap allows the detection of centred carbon and oxygen radicals, increasing their half-life [28]. For all compounds, one hyperfine pattern of six lines was obtained (Fig. 4). The hyperfine coupling constants were obtained by computer simulation of the spectra using WinSIM 9 [29].

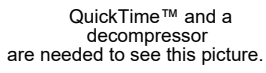

QuickTime ${ }^{\mathrm{TM}}$ and decompressor
are needed to see this picture

Fig. (3). Cyclic voltammograms for $1 \mathrm{mmol} / \mathrm{L}$ of compound 3 for $\mathrm{v}$ $=0.05-2.5 \mathrm{~V} / \mathrm{s}$.

Fig. (4). A) ESR experimental spectrum corresponding to the radical obtained from electrochemical oxidation of the stabilized compound 2. B) ESR simulated spectrum of compound 2, obtained using WinSIM. 
The coupling constants, $\mathrm{a}_{\mathrm{N}}$ and $\mathrm{a}_{\mathrm{H}} \approx 13.7$ and $\approx 2.2 \mathrm{G}$, were assigned to the nitrogen and the hydrogen atoms in position $\beta$ to the spin catcher, respectively. This hyperfine pattern is due to spin adduct formation of the coumarin radical trapped by PBN (Fig. 5).

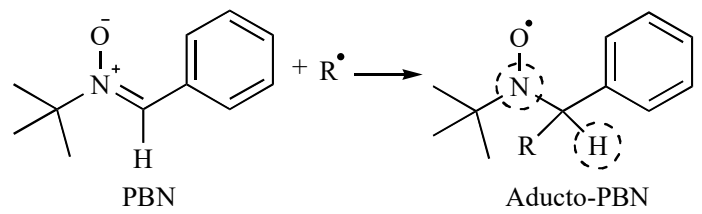

Fig. (5). Spin adduct formed with the spin trap PBN.

Based on these results, the oxidation mechanism for 3amidocoumarins was proposed (Fig. 6). Oxidation seems to occur on the nitrogen of the amide group and subsequent transposition of the hydrogen atom to form the centred carbon radical species, similar to that described by Sanchez et al. for symmetrical ferulic acid amide derivatives [30].<smiles>[CH2]C(=O)Nc1cc2ccccc2oc1=O</smiles>

Fig. (6). Proposed oxidation mechanism for compound 1.

The oxidation mechanism proposed for hydroxylated coumarins involves the formation of centred oxygen radicals and subsequent delocalization of the unpaired electron to generate a carbon centred radical (Fig. 7).

Oxygen radical absorbance capacity-fluorescein (ORACFL) index of all 3-amidocoumarins was determined. These values allow to evaluate the influence that the hydroxyl<smiles></smiles>

Fig. (7). Proposed oxidation mechanism for compound 5.

groups at position 4 of the coumarin may have on the antioxidant activity. Compounds 3 and 7 showed the best ORACFL indexes (Fig. 8). Based on the ORAC-FL values (Table 1), the antioxidant profile of the studied molecules can be organized as: $\mathbf{3}>\mathbf{7}>\mathbf{5}>\mathbf{6} \approx \mathbf{8} \approx \mathbf{4} \approx \mathbf{9}>\mathbf{2} \approx \mathbf{1}$. ORAC-FL indexes described here are similar to those previously determined for coumarins presenting the same structural patterns $[4,15]$. However, in general these values are lower than the determined for 3-aryl-4-hydroxycoumarins [14]. This could be explained considering the formation of an intramolecular hydrogen bond between the carbonyl group at position 3 and the hydroxyl group at position 4 .

Reactivity against radicals was studied by means of the spin-trapping methodology, which is based on the competitive reactions between the coumarin derivative and the spintrap (DMPO). The reactivity against a mixture of radical species for all of the studied coumarin derivatives was carried out by adapting the $\mathrm{H}_{2} \mathrm{O}_{2} / \mathrm{NaOH} / \mathrm{DMF} / \mathrm{DMPO}$ system using a non-catalytic and competitive Fenton reaction in which the spin trap DMPO and the antioxidant molecule compete for the hydroxyl, superoxide anion, and methyl radicals [31]. Four wide hyperfine lines were observed in the control measurement (labelled as blank), indicating the absence of tested molecules (Fig. 9). This happened because a mixture of DMPO- $\mathrm{OH}, \mathrm{DMPO}-\mathrm{CH}_{3}$, and DMPO- $\mathrm{O}_{2}{ }^{-{ }^{-}}$adducts was formed.

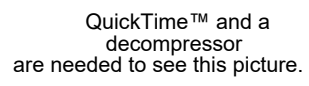

Fig. (8). A) ORAC-FL profile of compound 7. B) AUC as a function of the concentration of compound 7. 
Scavenging of the radical was calculated by adding a constant concentration of the corresponding derivative and measuring the decrease percentage in the integration of the signals regarding to the control solution. Using this method, it was observed that the intensity of the spectrum decreased in the presence of the tested compounds added to the system (grey line, Fig. 9).

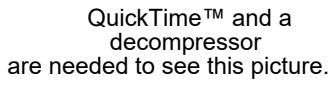

Fig. (9). ESR spectra of compound 5 and blank.

The same behaviour was observed for all derivatives. The scavenging values obtained for non-hydroxylated compounds can be related to the presence of a hydrolysed species with structural similarity to a hydroxycinnamic acid [32].

2',7'-Dichlorodihydrofluorescein diacetate $\left(\mathrm{DCFH}_{2}-\mathrm{DA}\right)$ has been extensively used as a probe for the detection of oxidative stress. It can reflect the overall oxidative profile of the cell. This dye diffuses passively through the cellular membrane and is transformed into $\mathrm{DCFH}_{2}-\mathrm{a}$ nonfluorescent compound which emits fluorescence when it is oxidized to 2,7-dichlorofluorescein (DCF) $\left(\lambda_{\text {excitation }}=498\right.$ $\mathrm{nm} ; \lambda_{\text {emission }}=522 \mathrm{~nm}$ ) by intracellular reactive oxygen species (ROS)- due to intracellular esterase activity [33].
The 2,2'-azo-bis(2-methylpropionamidine)dihydro chloride (AAPH) is usually employed as the generator of peroxyl radicals. It is generally accepted that hydrophilic antioxidants can interact more with the radicals generated by AAPH than hydrophobic antioxidants (Fig. 10A) [34].

RAW 264.7 cells were used in the evaluation of antioxidant activity of 3-aminocoumarins [7]. AAPH was used as stressor agent and $\mathrm{DCFH}_{2}$-DA as the detection system. The hydroxylated compounds showed cellular antioxidant activity related to lipophilicity systems in the following order: $7>$ $3>5$ (Fig. 10B). As higher the lipophilicity, as greater cellular antioxidant activity was found. This relationship is reversed for non-hydroxylated compounds, where the higher lipophilicity is related to a decrease in the antioxidant activity, possibly by improved interaction with the cell membrane.

After the electrochemical study, biological experiments were carried out to deeply explore the therapeutic potential of the studied series. Cytotoxicity of the synthesized coumarins was evaluated using mammalian cells (macrophages RAW 264.7 murine cells). The cells were exposed to each compound for 24 h. 3-(4,5-Dimethylthiazol-2-yl)-2,5diphenyltetrazolium bromide (MTT) assay was used to measure the response of the cells. The $\mathrm{IC}_{50}$ values were determined, and as desired, most of the studied derivatives proved to be non-cytotoxic against mammalian cells ( $p>$ 0.01 ). Compounds 8 and 9 showed the highest cytotoxicity values (Table 2). All compounds showed higher trypanocidal activity against both cellular forms of the parasite (\% cell viability, Table 2) than the previously described 2-chloro- $N$ (coumarin-3-yl)acetamide [4]. In addition, these compounds showed similar activity to a previously studied series of 3carboxamidocumarins [15]. Based on this data, the design of the new molecules can be considered a step forward in the use of 3-substituted coumarins as multi-target compounds against $T$. cruzi. These findings inspired us to deeply explore the mechanisms of action and the trypanocidal potential of the best compounds of the series.

The trypanocidal activity of substituted amidocoumarins 
Table 1. Results obtained in antioxidant homogeneous assays (oxidation potential, radical scavenging and ORAC-FL) and cellular antioxidant activity (CAA) assay.

\begin{tabular}{|c|c|c|c|c|c|}
\hline \multirow{2}{*}{ Compound } & \multirow{2}{*}{$\mathrm{E}_{\mathrm{pa}}(\mathrm{V}) \mathrm{Ag} / \mathrm{AgCl}$} & \multirow{2}{*}{ Radical Scavenging (\%) } & \multirow{2}{*}{ ORAC-FL } & \multicolumn{2}{|c|}{ Cellular Antioxidant Activity (\%) } \\
\hline & & & & $10 \mu \mathrm{M}$ & $1 \mu \mathrm{M}$ \\
\hline 1 & 1.09 & $23.6 \pm 1.2$ & $0.01 \pm 0.01$ & $53.02 \pm 1.09$ & $46.60 \pm 3.88$ \\
\hline 2 & 1.51 & $81.1 \pm 1.7$ & $0.02 \pm 0.01$ & $43.46 \pm 1.31$ & $31.76 \pm 1.48$ \\
\hline 3 & 1.14 & $84.3 \pm 1.1$ & $2.19 \pm 0.02$ & $39.35 \pm 3.13$ & $23.73 \pm 2.80$ \\
\hline 4 & 1.45 & $71.8 \pm 2.2$ & $0.07 \pm 0.02$ & $39.71 \pm 2.79$ & $25.40 \pm 2.12$ \\
\hline 5 & 1.16 & $95.6 \pm 1.5$ & $0.65 \pm 0.03$ & $34.48 \pm 1.88$ & $21.34 \pm 2.72$ \\
\hline 6 & 1.40 & $80.1 \pm 1.8$ & $0.08 \pm 0.02$ & $36.07 \pm 1.81$ & $22.12 \pm 2.15$ \\
\hline 7 & 1.12 & $83.3 \pm 2.1$ & $1.06 \pm 0.03$ & $42.06 \pm 2.46$ & $23.58 \pm 2.81$ \\
\hline 8 & 1.43 & $26.8 \pm 1.9$ & $0.08 \pm 0.01$ & $33.28 \pm 2.87$ & $18.62 \pm 2.17$ \\
\hline 9 & 1.52 & $32.7 \pm 1.3$ & $0.06 \pm 0.02$ & $30.78 \pm 2.69$ & $21.43 \pm 1.87$ \\
\hline Trolox & 0.18 & $\mathbf{3 0 . 3} \pm 1.6$ & $1.00 \pm 0.20$ & -- & -- \\
\hline
\end{tabular}

Table 2. In vitro cytotoxicity against murine RAW 264.7 macrophages $\left(\right.$ IC $\left._{50}\right)$, and trypanocidal activity against epimastigote and trypomastigote stages.

\begin{tabular}{|c|c|c|c|}
\hline \multirow{2}{*}{ Compound } & \multirow{2}{*}{$\begin{array}{c}\text { Cytotoxicity RAW 264.7 } \\
\left(\mathbf{I C}_{\mathbf{5 0}} \boldsymbol{\mu M}\right)\end{array}$} & Epimastigotes & Tell Viability \\
\cline { 2 - 4 } & $>100$ & $53.66 \pm 2.64$ & $46.34 \pm 2.66$ \\
\hline \hline $\mathbf{1}$ & $80.12 \pm 1.18$ & $23.08 \pm 2.39$ & $12.42 \pm 2.28$ \\
\hline $\mathbf{3}$ & $>100$ & $34.96 \pm 0.72$ & $53.48 \pm 2.76$ \\
\hline $\mathbf{4}$ & $>100$ & $47.99 \pm 1.75$ & $50.18 \pm 1.34$ \\
\hline $\mathbf{5}$ & $>100$ & $51.68 \pm 1.81$ & $54.03 \pm 2.79$ \\
\hline $\mathbf{6}$ & $>100$ & $33.21 \pm 2.20$ & $49.63 \pm 1.66$ \\
\hline $\mathbf{7}$ & $83.40 \pm 2.26$ & $48.72 \pm 2.93$ & $42.28 \pm 2.15$ \\
\hline $\mathbf{8}$ & $62.04 \pm 2.05$ & $59.86 \pm 3.03$ & $55.31 \pm 4.16$ \\
\hline $\mathbf{9}$ & $12.20 \pm 1.29$ & $27.95 \pm 2.01$ & $23.63 \pm 3.30$ \\
\hline $\mathbf{N F}$ & $>100$ & $10.04 \pm 0.22$ & $17.82 \pm 2.52$ \\
\hline
\end{tabular}

against the epimastigote and trypomastigote stages of $T$. cruzi was determined. The viability of epimastigote and trypomastigote forms of $T$. cruzi was determined using solutions of $100 \mu \mathrm{M}$ of each coumarin (Table 2). In general, nonhydroxylated derivatives have greater trypanocidal activity against both cell stages. However, compounds 2 and 7 proved to be more active against the trypomastigote form than the epimastigote one.

The best compounds of the studied series proved to be compound $\mathbf{2}$ and $\mathbf{6}$, presenting compound $\mathbf{2}$ higher trypanocidal activity than NFX against the epimastigote stage. Despite of having the second-best activity profile of the series, compound 9 was discarded as a potential trypanocidal agent due to the high cytotoxicity against mammalian cells. No general direct correlation was found between the cellular antioxidant activity and the trypanocidal activity against the epimastigote form of T. cruzi (Fig. 11).

At the cellular level, there is no strong influence in the antioxidant activity of the hydroxyl group at position 4 . Therefore, the effect of the antioxidant capacity in the trypanocidal activity of these compounds is discarded. Moreover, the effect on mitochondrial membrane potential of 3carboxamidocoumarins was described, being a possible intracellular explanation for the activity of the tested compounds [15]. Also, some coumarins have been described to inhibit the enzyme GAPDH, a key protein in the glycolytic pathway of trypanosomatids $[3,35]$. In addition, being able to generate oxidative stress in the parasite $T$. cruzi, which is deficient in antioxidant mechanisms (enzymatic and non- 


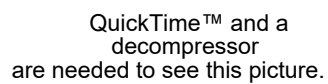

are needed to see this picture.
Fig. (11). Relationship between cellular antioxidant and trypanocidal activities, against epimastigote stage of $T$. cruzi, in the presence of 3-amidocoumarins. Circle - compound with major trypanocidal activity.

enzymatic) comparing to mammalian cells, is an excellent strategy to improve the trypanocidal activity [36].

To understand the possible mechanism of action of the studied series, the formation of radical species in the epimastigote form of $T$. cruzi was determined. The most interesting compounds were $\mathbf{2}$ and $\mathbf{6}$, which presented the highest trypanocidal activity. Likewise, hydroxyl-analogues $\mathbf{3}$ and $\mathbf{7}$ were also evaluated.

To perform the free radical detection in the parasitic medium, ESR spectroscopy was employed, using the technique of spin trapping. The spin-trap was DMPO, that can cross cell membranes and react with several centred carbon and oxygen free radicals [37-39]. A solution of DMSO was used as a control (no radical species were detected). In this assay, the compounds were used in a concentration of $5 \mathrm{mM}$ (Fig. 12). Non-hydroxylated compounds generated the same spectral pattern after $10 \mathrm{~min}$ of incubation at $28^{\circ} \mathrm{C}$. The strong intensity of the observed signals is consistent with the trapping of an oxygen-centred radical $(\mathrm{aN}=\mathrm{aH} \sim 14.86 \mathrm{G})$. Likewise, a triplet $(\mathrm{aN} \sim 15.46 \mathrm{G})$ was observed in the spectrum corresponding to a paramagnetic compound oxidized derivative of DMPO (DMPOX, 5,5-dimethyl-2-oxopyrrolin-1-oxyl). The hydroxylated compounds generated the same spectral pattern. A quartet splitting pattern $(\mathrm{aN} \sim \mathrm{aH} \sim 14.87 \mathrm{G})$, due to the DMPO-OH radical adduct, was observed. In addition, a sextet corresponding to the entrapment ( $\mathrm{aN} \sim 16.40 \mathrm{G}$ aH $\sim 23.20 \mathrm{G}$ ) was generated, as described by Robelo-O'Ryan et al. [40].

These results may indicate that the mechanism of action of these coumarins could be initiated through the generation of ROS (Fig. 12). A possible pathway would be the oxidative stress. The intensity of the signals indicates that hydroxylated coumarins generate reactive oxygen and carbon species in lower concentration than non-hydroxylated coumarins. In addition, the ESR spectrum in the presence of menadione (generator oxidative stress) shows a hyperfine pattern corresponding to the DMPOX, corroborating the generation of oxidative stress in the parasite.
Fig. (12). Spectra of the spin-adducts generated in epimastigote forms of T. cruzi (Dm28c) at room temperature. A) Spectrum recorded with epimastigotes incubated with compound $\mathbf{2}$ and DMPO. B) Spectrum recorded with epimastigotes incubated with compound 3 and DMPO. C) Spectrum recorded with epimastigotes incubated with menadione and DMPO. D) Spectrum of the control (DMSO). Hyperfine pattern corresponding to the spin-adduct DMPO-OH have been marked with $(\mathrm{x})$. The hashtag $(\#)$ indicates the adduct between a carbon-centred radical and DMPO, and $(+)$ indicates the DMPOX adduct.

Taking into account the activity observed, $\mathrm{IC}_{50}$ of compounds 2, 3, 6 and 7 against $T$. cruzi trypomastigote form were determined (Table 3). Compound $2\left(\mathrm{IC}_{50}=21.53 \mu \mathrm{M}\right)$ presented an activity and a selectivity index like NFX. This data corroborates the potential of this compound as an interesting trypanocidal agent.

Table 3. In vitro cytotoxicity of compounds $2,3,6$ and 7 against trypomastigote stages of $T$. cruzi.

\begin{tabular}{|c|c|c|}
\hline Compound & $\mathbf{I C}_{\mathbf{5 0}} \boldsymbol{\mu M}$ & SI* $^{*}$ \\
\hline \hline $\mathbf{2}$ & $21.53 \pm 1.21$ & 3.72 \\
\hline $\mathbf{3}$ & $36.41 \pm 2.11$ & 2.75 \\
\hline $\mathbf{6}$ & $52.12 \pm 1.71$ & 1.92 \\
\hline $\mathbf{7}$ & $56.92 \pm 1.53$ & 1.47 \\
\hline $\mathbf{N F X}$ & $22.56 \pm 1.03$ & 4.43 \\
\hline
\end{tabular}

* Selectivity Index = $\mathrm{IC}_{50}$ macrophages RAW 264.7//C 50 parasite. 
The determined $\mathrm{IC}_{50}$ values indicate that there is no strong influence of the hydroxyl groups in the trypanocidal activity of 3-amidocoumarin derivatives. It seems that the substituent on the amide group is the main factor to modulate the trypanocidal activity of the studied compounds. In general, aromatic heterocyclic groups decreased the trypanocidal activity. These results give interesting information about the structural properties and the trypanocidal activity for the design of new 3-amidocoumarins.

The mitochondria of T. cruzi is fundamental for the survival of the parasite and for the regulation of processes related to the energy balance and modulation of apoptosis. A crucial point in mitochondrial metabolism is the consumption of oxygen, an important step of oxidative phosphorylation within the mitochondrial matrix. Therefore, the effect on the mitochondrial function of a series of 3-carboxamidocoumarins was studied [15]. To determine a possible target of these compounds, oxygen consumption was determined for the 4 substituted amidocoumarins that showed the best activities against epimastigotes of T. cruzi (Fig. 13). Finding that there was no alteration in oxygen consumption of epimastigotes, it was proved that there is no effect on the mitochondrial matrix, and a different trypanocidal mechanism of action is involved in the effect of the studied coumarins. tion (Sigma-Aldrich). The described derivatives were efficiently synthesized according to the protocols outlined in Scheme 1 [17-22].

\subsection{Evaluation of Redox Properties}

CV was carried out using a Metrohm 797 VA instrument with a 797 VA Stand convertor and a 797 VA Processor, in $\mathrm{DMF}$, at room temperature, with tetrabutylammonium perchlorate (TBAP) $0.1 \mathrm{M}$ as supporting electrolyte, using a three-electrode cell. A glassy carbon electrode presenting an area of $0.03 \mathrm{~cm}^{2}$ was used as the working electrode, a platinum wire as the auxiliary electrode and silver/silver chloride $(\mathrm{Ag} / \mathrm{AgCl}, 3 \mathrm{M} \mathrm{KCl})$ as the reference electrode. DPV method was carried out under the same experimental conditions, with potential sweeps between 0.0 and $1.7 \mathrm{~V}$ and a scan velocity of $20 \mathrm{mV} / \mathrm{s}$. The pulse amplitude was $50 \mathrm{mV}$ during $0.5 \mathrm{~s}$, and step height was $4 \mathrm{mV}$.

\subsection{Monitoring of Electrochemically Generated Radicals by ESR}

ESR spectra were recorded in the $\mathrm{X}$ band $(9.85 \mathrm{GHz})$ using a Bruker ECS106 spectrometer with a rectangular cavity and $50-\mathrm{kHz}$ field modulation. The hyperfine splitting constants were estimated to be accurate within $0.05 \mathrm{G}$. The radicals were generated by an in situ electrolytic oxidation process under the same experimental conditions as those of DPV. Every spectrum was obtained after 100 scans. The radicals were trapped by adding $200 \mathrm{mM}$ of $N$-tert-butyl- $\alpha$ phenyl-nitrone $(\mathrm{PBN})$. Electron spin resonance (ESR) spectra were simulated using the program EPR-WinSIM Version 0.98 .

\subsection{Antioxidant Capacity}

\subsubsection{Oxygen Radical Antioxidant Capacity-fluorescein (ORAC-FL)}

The ORAC-FL analysis was carried out on a EnSpire multimode plate reader from Perkin-Elmer, using white polystyrene 96-well plates, purchased from Nunc (Denmark). Fluorescence was read from the top, with an excitation of $485 \mathrm{~nm}$ and emission of $528 \mathrm{~nm}$. The reaction was carried out in $75 \mathrm{mM}$ sodium phosphate buffer ( $\mathrm{pH} 7.4$ ), $200 \mu \mathrm{L}$ final volume. FL (40 nM, final concentration) and coumarin solutions in methanol (range of concentration between 0.3 $\mu \mathrm{M}$ to $2 \mu \mathrm{M}$ ) were placed in each well of 96 -well plate. The mixture was pre-incubated for $15 \mathrm{~min}$ at $37^{\circ} \mathrm{C}$, before rapidly adding the AAPH solution (18 mM, final concentration). The microplate was immediately placed in the reader and automatically shaken prior to each reading. The fluorescence was recorded every $1 \mathrm{~min}$ for $180 \mathrm{~min}$. A blank with FL and AAPH using methanol instead of the antioxidant solution was used in each assay. Five calibration solutions using Trolox $(0.5 \mu \mathrm{M}$ to $2.5 \mu \mathrm{M})$ as antioxidant were also used in each assay. The inhibitory capacity was expressed as ORACFL values and it was quantified by integration of the area under the curve $\left(\mathrm{AUC}_{\mathrm{NET}}\right)$. All reaction mixtures were prepared in triplicate and at least three independent assays were performed for each sample. The area under the fluorescence decay curve (AUC) was calculated integrating the decay of the fluorescence where $F_{0}$ is the initial fluorescence read at 0 $\min$ and $\mathrm{F}$ is the fluorescence read at time. The net AUC 
corresponding to the sample was calculated by subtracting the AUC corresponding to the blank. Data processing was performed using Origin Pro 8.5 SR2 (Origin Lab Corporation, USA). The ORAC-FL indexes were calculated according to the following equation (1):

$$
\begin{aligned}
\text { ORAC ĞFLindex }= & \frac{\left(A U C_{A H} \breve{\mathrm{G}} A U_{\text {CONTROL }}\right)}{\left(A U C_{A H} \breve{\mathrm{G}} A U_{\text {CONTROL }}\right)} \times \\
& \frac{(T R O L O X)}{(A H)}
\end{aligned}
$$

\subsubsection{Evaluation of $\mathrm{OH}$ Scavenging by ESR}

ESR spectra were recorded in the X band $(9.7 \mathrm{GHz})$ using a Bruker ECS 106 spectrometer with a rectangular cavity and $50-\mathrm{kHz}$ field modulation, equipped with a highsensitivity resonator at room temperature. Spectrometer conditions were: microwave frequency $9.81 \mathrm{GHz}$; microwave power $20 \mathrm{~mW}$; modulation amplitude $0.91 \mathrm{G}$; receiver gain $59 \mathrm{db}$; time constant $81.92 \mathrm{msec}$; and conversion time 40.96 msec. Scavenging activity of each derivative was estimated by comparing the DMPO-OH adduct signals in the antioxidant-radical reaction mixture and the control, at the same reaction time, and was expressed as scavenging of hydroxyl radical percent. Compounds reactivity against radical peroxide, using the non-catalytic Fenton type method, was investigated [31]. Samples were prepared as follows: $100 \mu \mathrm{L}$ of DMF and $50 \mu \mathrm{L}$ of $\mathrm{NaOH}(25 \mathrm{mM})$ were mixed, followed by the addition of $50 \mu \mathrm{L}$ of DMPO ( $30 \mathrm{mM}$ final concentration), sample $50 \mu \mathrm{L}(20 \mathrm{mM}$ in DMF) and finally $50 \mu \mathrm{L}$ of hydrogen peroxide $(30 \%)$. The mixture was put into ESR cell and the spectrum was recorded after $5 \mathrm{~min}$ of reaction.

\subsubsection{Evaluation of Antioxidant Activity in RAW 264.7 Cells}

Cellular antioxidant activity (CAA) was evaluated in RAW 264.7 cells using 2',7'-dichlorodihydrofluorescein diacetate $\left(\mathrm{DCFH}_{2}-\mathrm{DA}\right)$ as fluorescent probe. The cells were plated in white sterile polystyrene flat-bottom 96-well microplates (Nunc, Denmark) at a concentration of 50,000 cells per well and incubated for $24 \mathrm{~h}$ at $37^{\circ} \mathrm{C}$ and $5 \% \mathrm{CO}_{2}$ in RPMI 1640 culture medium. The cells were washed with $150 \mathrm{~mL}$ of PBS, $\mathrm{pH}=7.4$ and incubated for $1 \mathrm{~h}$ with $100 \mu \mathrm{L}$ of RPMI 1640 containing $25 \mathrm{mM}$ of $\mathrm{DCFH}_{2}$-DA. The compounds were added at final concentrations of 1 and $10 \mu \mathrm{M}$. After $1 \mathrm{~h}$ incubation, the medium was discarded, and the cells were gently washed twice with $200 \mu \mathrm{L}$ of PBS. Then they were incubated with AAPH at a final concentration of $600 \mathrm{mM}$ in phosphate buffered saline (PBS). Fluorescence was measured immediately after AAPH addition in a EnSpire from Perkin-Elmer in 96-well plates at $37^{\circ} \mathrm{C}$ using an excitation of $485 \mathrm{~nm}$ and an emission of $538 \mathrm{~nm}$. The evaluation was carried out every min for $1 \mathrm{~h}$ and the CAA values were calculated by the equation (2) [41]:

$$
C A A \%=\left(\frac{\Delta F \breve{\mathrm{G}} \Delta F_{A H}}{\Delta F}\right) \times 100
$$

$F=$ fluorescence intensity in the presence of free radical, without phenolic compounds. $F_{\mathrm{AH}}=$ fluorescence intensity in the presence of free radical and phenolic compounds. All measures were done at the same period.

\subsubsection{Evaluation of the Cytotoxic and Trypanocidal Activity}

\subsubsection{Cytotoxicity Assay}

The effect of compound treatments on RAW 264.7 cells was evaluated through the tetrazolium dye MTT assay as a viability test [42]. The compounds under study, dissolved in DMSO, were added to the culture media at concentrations between 10-100 $\mu \mathrm{M}$.

$10 \mu \mathrm{L}$ of $5 \mathrm{mg} / \mathrm{mL}$ MTT plus $0.22 \mathrm{mg} / \mathrm{mL}$ phenazine metosulfate (electron carrier) were added at each well containing RAW 264.7 cell culture in $100 \mu \mathrm{L}$ RPMI 1640 without phenol red. DMSO final concentration was less than $0.1 \% \mathrm{v} / \mathrm{v}$. After incubation for $4 \mathrm{~h}$ at $37^{\circ} \mathrm{C}$, the generated water-insoluble formazan dye was dissolved by the addition of $100 \mu \mathrm{L}$ of $10 \% \mathrm{w} / \mathrm{v}$ sodium dodecyl sulfate (SDS) in 0.01 $\mathrm{M} \mathrm{HCl}$. The plates were further incubated overnight at $37^{\circ} \mathrm{C}$, and optical density of the wells was determined using a microplate reader (Asys Expert Plus ${ }^{\mathcal{O}}$, Asys Hitachi, Austria) at $570 \mathrm{~nm}$. Under these conditions, the optical density is directly proportional to the viable cell number in each well. All experiments were performed at least three times and data reported as means and their standard deviations from triplicate cultures. Results are reported as $\mathrm{IC}_{50}$, regarding the control (cells in culture medium).

\subsubsection{Epimastigote Stage Viability Study}

Trypanocidal activity was evaluated against the $T$. cruzi epimastigote stage (clone Dm28c). It was measured by the MTT assay using $0.22 \mathrm{mg} \mathrm{mL}^{-1}$ phenazine metosulfate (as an electron carrier) [43]. T. cruzi epimastigotes (Dm28c strain), from the authors own collection (Programa de Farmacología Molecular y Clínica, Facultad de Medicina, Universidad de Chile, Santiago, Chile) were grown at $28^{\circ} \mathrm{C}$ in Diamond's monophasic medium, as reported earlier, but replacing blood with $4 \mu \mathrm{M}$ hemin. Fetal calf serum was added to a final concentration of $5 \%$. In this colorimetric assay for testing the trypanocidal activity, the coumarin derivatives were dissolved in DMSO and were added to $3 \times 10^{6}$ parasites $\mathrm{mL}^{-1}$ at $10 \mu \mathrm{M}$ final concentrations in RPMI 1640 culture medium for $24 \mathrm{~h}$, at $28^{\circ} \mathrm{C}$. DMSO final concentration was less than $0.1 \% \mathrm{v} / \mathrm{v}$. Likewise, NFX was added as a positive control. Tetrazolium salt was added at a final concentration of $0.5 \mathrm{mg}$ $\mathrm{mL}^{-1}$, incubated at $28{ }^{\circ} \mathrm{C}$ for $4 \mathrm{~h}$ and then solubilized with $10 \% \mathrm{SDS} / 0.1 \mathrm{mM} \mathrm{HCl}$ and incubated overnight. After incubation, the number of viable parasites was determined by absorbance measures at $570 \mathrm{~nm}$ in a multiwell plate reader (Asys Expert Plus). Untreated parasites were used as controls ( $100 \%$ of viability). Results are reported as the percentage of viable epimastigotes regarding the control (parasite in culture medium).

\subsubsection{Trypomastigote Stage Viability Study}

Vero cells were infected with Dm28c trypomastigotes at a 1:3 (cell:parasite) ratio. T. cruzi trypomastigotes were initially obtained from primary cultures of peritoneal macrophage from chagasic mice. Vero cells were cultured in 5\% fetal bovine serum supplemented RPMI 1640 medium in humidified air with $5 \% \mathrm{CO}_{2}$ at $37^{\circ} \mathrm{C}$. Vero cell cultures were 
then infected with trypomastigotes and incubated at $37^{\circ} \mathrm{C}$ in humidified air and 5\% $\mathrm{CO}_{2}$ for 5-7 days. After that time, the culture medium was collected, centrifuged at $500 \times \mathrm{g}$ for 5 $\mathrm{min}$, and the trypomastigote-containing pellet was resuspended in free-serum RPMI 1640 and penicillinstreptomycin at a final density of $1 \times 10^{7}$ parasites $/ \mathrm{mL}$. Trypomastigote viability assays were performed using the MTT reduction method as described previously. $1 \times 10^{7}$ parasites/ $\mathrm{mL}$ were incubated in free-serum RPMI 1640 culture medium at $37^{\circ} \mathrm{C}$, over $24 \mathrm{~h}$ with or without the studied compounds. An aliquot of the parasite suspension was extracted and incubated in a 96-well flat-bottom plate and MTT was added at a final concentration of $0.5 \mathrm{mg} / \mathrm{mL}$ using 0.22 $\mathrm{mg} / \mathrm{m}$ phenazine metosulfate (as an electron carrier), incubated at $28^{\circ} \mathrm{C}$ during $4 \mathrm{~h}$ and then made soluble with $10 \%$ SDS- $0.1 \mathrm{mM} \mathrm{HCl}$ and incubated overnight. Formazan formation was measured at $570 \mathrm{~nm}$, with a reference wavelength at $690 \mathrm{~nm}$, in a multiwell plate reader (Asys Expert Plus). Untreated parasites were used as controls (100\% viability). Results are reported as the percentage of viable parasites regarding the control (parasite in culture medium). In addition, the $\mathrm{IC}_{50}$ was determined.

\subsubsection{Detection of Radical Species in Parasitic Medium $\underline{\text { by ESR }}$}

T. cruzi epimastigotes (Dm28c), from our collection, were grown at $28{ }^{\circ} \mathrm{C}$ in Diamond's monophasic medium as reported earlier, with blood replaced by $4 \mathrm{mM}$ hemin [44]. Fetal calf serum was added to a final concentration of $4 \%$. Parasites: $8 \times 10^{7}$ cells correspond to $1 \mathrm{mg}$ protein or $12 \mathrm{mg}$ of fresh weight. ESR spectra were produced using parasite in epimastigote stage, in a reaction medium containing $1 \mathrm{mM}$ NADPH and $100 \mathrm{mM}$ DMPO, in $20 \mathrm{mM}$ phosphate buffer, $\mathrm{pH}$ 7.4. All experiments were done after of $10 \mathrm{~min}$ of incubation, at $28^{\circ} \mathrm{C}$, for each 3 -amidocoumarin $(5 \mathrm{mM})$ with epimastigotes of T. cruzi, NADPH and DMPO, in an aerobic environment.

\subsubsection{Oxygen Consumption Determination in Epi- mastigotes of T. cruzi}

The rates of oxygen consumption were polarographically measured at $28^{\circ} \mathrm{C}$ with a No. 5331 Clark-type electrode (Yellow Springs Instrument, Yellow Spring, OH, USA) using an YSI model 5300 monitor (Yellow Springs Instrument, Yellow Spring, OH, USA) linked to a DI-148U data acquisition module with a USB interface. The data were acquired with Windaq Acquisition Waveform Recorder software (DataQ Instruments, USA) [45, 46].

A concentration of $1 \mathrm{mg}$ of protein equivalent to $8.0 \times 10^{7}$ of epimastigotes Dm28c was used (PBS buffer, $\mathrm{pH}=7.4$ ). The effect of the treatment was observed during $8 \mathrm{~min}$ and the concentration of the compounds was equal to the $\mathrm{IC}_{50}$. The response in the consumption of oxygen was expressed by the percentage of the consumption regarding the control.

\subsection{Statistical Analysis}

Statistical analysis was performed using Graph Pad Prism 4.03 (GraphPad Software. San Diego. California USA). Data are expressed as mean $\pm \mathrm{SD}$ of three independent experiments. $\mathrm{IC}_{50}$ values were calculated from the dose-response curves (MTT assays) obtained by nonlinear curve fitting.
Statistical analysis was performed using one-way ANOVA with Dunnett post-test. Data are considered statistically significant when $\mathrm{p}<0.05$.

\section{CONCLUSION}

The obtained results showed that compound 2 can be an alternative to NFX against trypomastigote form of T. cruzi, displaying similar activity in the low micromolar range, and being non-toxic to mammal cells. In addition, results showed that the studied series of compounds can be electrochemically oxidised through an irreversible mechanism, leading to radical species which polymerize on the surface of the glassy carbon electrode. The influence of the hydroxyl groups at position 4 was also studied. Surprisingly, this has no representative effect against the different radical species. To determine a possible mechanism of action, the generation of radical species within the parasite was determined, evidencing that the oxidative stress is involved in the activity. However, the effect of these compounds on the oxygen uptake of T. cruzi parasites was discarded. Based on the experimental assays, compound $\mathbf{2}$ can be considered for lead optimization and the design of new and more effective ans saffer trypanocidal compounds.

\section{ETHICS APPROVAL AND CONSENT TO PARTICI- PATE}

Not applicable.

\section{HUMAN AND ANIMAL RIGHTS}

No Animals/Humans were used for studies that are the basis of this research.

\section{CONSENT FOR PUBLICATION}

Not applicable.

\section{CONFLICT OF INTEREST}

The authors declare no conflict of interest, financial or otherwise.

\section{ACKNOWLEDGEMENTS}

The authors thank PhD fellowship from CONICYT, MMB grant for operational expenses ( $\left.\mathrm{N}^{\circ} 21150192\right)$, ML Post-PhD/FONDECYT N³160022, JDM FONDECYT $\mathrm{N}^{\circ} 1130189$, COA FONDECYT 1150175 and Postdoctoral fellowship from Xunta da Galicia (ED481B 2014/086-0).

\section{REFERENCES}

[1] Nunes, M.C.P.; Dones, W.; Morillo, C.A.; Encina, J.J.; Ribeiro, A.L. Chagas disease: an overview of clinical and epidemiological aspects. J. Am. Coll. Cardiol., 2013, 62(9), 767-776.

[2] Ferreira, L.G.; Andricopulo, A.D. Drug repositioning approaches to parasitic diseases: a medicinal chemistry perspective. Drug Discov. Today., 2016, 21(10), 1699-1710.

[3] Freitas, R.F.; Prokopczyk, I.M.; Zottis, A.; Oliva, G.; Andricopulo, A.D.; Trevisan, M.T.; Vilegas, W.; Silva, M.G.; Montanari, C.A. Discovery of novel Trypanosoma cruzi glyceraldehyde-3phosphate dehydrogenase inhibitors. Bioorg. Med. Chem., 2009, 17(6), 2476-2482.

[4] Figueroa, R.; Matos, M.J.; Vazquez-Rodriguez, S.; Santana, L.; Uriarte, E.; Olea-Azar, C.; Maya, J.D. Synthesis and evaluation of 
antioxidant and trypanocidal properties of a selected series of coumarin derivatives. Future Med. Chem., 2013, 5(16), 1911-1922.

[5] de Oliveira, T.B.; Pedrosa, R.C.; Filho, D.W. Oxidative stress in chronic cardiopathy associated with Chagas disease. Inter. J. Cardiol., 2007, 116(3), 357-363.

[6] Gupta, S.; Wen, J.-J.; Garg, N.J. Oxidative Stress in Chagas Disease. Interdiscip. Perspect. Infect. Dis., 2009, 2009, 190354.

[7] Rodríguez-Angulo, H.; García, O.; Castillo, E.; Cardenas, E.; Marques, J.; Mijares, A. Etanercept induces low QRS voltage and autonomic dysfunction in mice with experimental Chagas disease. Arq. Bras. Cardiol., 2013, 101(3), 205-210.

[8] Kobo, P.I.; Ayo, J.O.; Aluwong, T.; Zezi, A.U.; Maikai, V.; Ambali, S.F. Flavonoid mixture ameliorates increase in erythrocyte osmotic fragility and malondialdehyde concentration induced by Trypanosoma brucei brucei-infection in Wistar rats. Res. Vet. Sci., 2014, 96(1), 139-142.

[9] Stanchev, S.; Hadjimitova, V.; Traykov, T.; Boyanov, T.; Manolov, I. Investigation of the antioxidant properties of some new 4hydroxycoumarin derivatives. Eur. J. Med. Chem., 2009, 44(7), 3077-3082.

[10] Kontogiorgis, C.A.; Hadjipavlou-Litina, D.J. Synthesis and biological evaluation of novel coumarin derivatives with a 7-azomethine linkage. Bioorg. Med. Chem. Lett., 2004, 14(3), 611-614.

[11] Symeonidis, T.; Fylaktakidou, K.C.; Hadjipavlou-Litina, D.J.; Litinas, K.E. Synthesis and anti-inflammatory evaluation of novel angularly or linearly fused coumarins. Eur. J. Med. Chem., 2009, 44(12), 5012-5017.

[12] Chimenti, F.; Secci, D.; Bolasco, A.; Chimenti, P.; Granese, A.; Befani, O.; Turini, P.; Alcaro, S.; Ortuso, F. Inhibition of monoamine oxidases by coumarin-3-acyl derivatives: biological activity and computational study. Bioorg. Med. Chem. Lett., 2004, 14(14), 3697-3703.

[13] Lin, H.-C.; Tsai, S.-H.; Chen, C.-S.; Chang, Y.C.; Lee, C.M.; Lai, Z.Y.; Lin, C.M. Structure-activity relationship of coumarin derivatives on xanthine oxidase-inhibiting and free radical-scavenging activities. Biochem. Pharmacol., 2008, 75(6), 1416-1425.

[14] Pérez-Cruz, F.; Serra, S.; Delogu, G.; Lapier, M.; Maya, J.D.; OleaAzar, C.; Santana, L.; Uriarte, E Antitrypanosomal and antioxidant properties of 4-hydroxycoumarins derivatives. Bioorg. Med. Chem. Lett., 2012, 22(17), 5569-5573.

[15] Muñoz, A.; Fonseca, A.; Matos, M.J.; Uriarte, E.; Santana, L.; Borges, F.; Figueroa, R.; Olea Azar, C. Evaluation of antioxidant and antitrypanosomal properties of a selected series of synthetic 3carboxamidocoumarins. ChemistrySelect., 2016, 1(15), 4957-4964.

[16] Salomão, K.; De Santana, N.A.; Molina, M.T.; De Castro, S.L.; Menna-Barreto, R.F.S. Trypanosoma cruzi mitochondrial swelling and membrane potential collapse as primary evidence of the mode of action of naphthoquinone analogues. BMC Microbiol., 2013, 13(1), 196-208.

[17] Linch, F.W. CLXXXVI.-The action of sodium hypobromite on carbamide derivatives. Part I. J. Chem. Soc. Transactions., 1912, 101, 1755-1758.

[18] Matos, M.J.; Vilar, S.; Kachler, S.; Celeiro, M.; VazquezRodriguez, S.; Santana, L.; Uriarte, E.; Hripcsak, G.; Borges, F.; Klotz, K.-N. Development of novel adenosine receptor ligands based on the 3-amidocoumarin scaffold. Bioorg, Chem., 2015, 61, $1-6$.

[19] Okumura, K. Studies on novobiocin and related compounds. I syntheses of 3-acylamino-4-hydroxycoumarin derivatives. Yakugaku Zasshi., 1960, 80(4), 525-532.

[20] Klussmann, E.; Rosenthal, W.; Rademann, J.; Christian, F.; Meyer, S. Non-peptidic inhibitors of akap/pka interaction. Canadian Intellectual Property Office. 2006.

[21] Viña, D.; Matos, M.J.; Yañez, M.; Santana, L.; Uriarte, E. 3Substituted coumarins as dual inhibitors of $\mathrm{AChE}$ and MAO for the treatment of Alzheimer's disease. Med. Chem. Comm., 2012, 3(2), 213-218.

[22] Marrian, D.H.; Russell, P.B.; Todd, A.R. The potato eelworm hatching factor. 6. Attempts to prepare artificial hatching agents. Part II. Some active arylidene- $\Delta(\beta: \gamma)$-butenolides and related compounds. Biochem. J., 1949, 45(5), 533-537.

[23] Kissinger, P.T.; Heineman, W.R. Cyclic voltammetry. J. Chem. Educ., 1983, 60(9), 702-706.
[24] Rodríguez, J.; Olea-Azar, C.; Barriga, G.; Folch, C.; Gerpe, A.; Cerecetto, H.; González, M. Comparative spectroscopic and electrochemical study of nitroindazoles: 3-Alcoxy, 3-hydroxy and 3oxo derivatives. Spectrochim. Acta A Mol. Biomol. Spectrosc., 2008, 70(3), 557-563.

[25] Nicholson, R.S.; Shain, I. Theory of stationary electrode polarography. Single scan and cyclic methods applied to reversible, irreversible, and kinetic systems. Anal. Chem., 1964, 36(4), 706-723.

[26] Hu, X.-R.; He, J.-B.; Wang, Y.; Zhu, Y.-W.; Tian, J.-J. Oxidative spectroelectrochemistry of two representative coumarins. Electrochim. Acta., 2011, 56(7), 2919-2925.

[27] Davies, M.J. Detection and characterisation of radicals using electron paramagnetic resonance (EPR) spin trapping and related methods. Methods., 2016, 109, 21-30.

[28] Pérez-Cruz, F.; Villamena, F.A.; Zapata-Torres, G.; Das, A.; Headley, C.A.; Quezada, E.; Lopez-Alarcon, C.; Olea-Azar, C. Selected hydroxycoumarins as antioxidants in cells: physicochemical and reactive oxygen species scavenging studies. J. Phys. Org. Chem., 2013, 26(10), 773-783.

[29] Duling, D.R. Simulation of multiple isotropic spin-trap EPR spectra. J. Magn. Reson. B., 1994, 104(2), 105-110.

[30] Sánchez, A.; Guillén-Villar, R.C.; Sánchez, R. Vargas, R.; Garza, J.; Matus, M.H.; Salas-Reyes, M.; Domínguez, Z. Electrochemical oxidation of symmetrical amides of ferulic acid in aprotic medium. Electrochim. Acta., 2014, 133, 546-554.

[31] Yoshimura, Y.; Inomata, T.; Nakazawa, H.; Kubo, H.; Yamaguchi, F.; Ariga, T. Evaluation of free radical scavenging activities of antioxidants with an $\mathrm{H}_{2} \mathrm{O}_{2} / \mathrm{NaOH} / \mathrm{DMSO}$ system by electron spin resonance. J. Agric. Food Chem., 1999, 47(11), 4653-4656.

[32] Pino, E.; Campos, A.M.; López-Alarcón, C.; Aspée, A.; Lissi, E. Free radical scavenging capacity of hydroxycinnamic acids and related compounds. J. Phys. Org. Chem., 2006, 19(11), 759-764.

[33] Mura, F.; Silva, T.; Castro, C.; Borges, F.; Zuñiga, M.C.; Morales, J.; Olea-Azar, C. New insights into the antioxidant activity of hydroxycinnamic and hydroxybenzoic systems: spectroscopic, electrochemistry, and cellular studies. Free Radic. Res., 2014, 48(12), 1473-1484.

[34] Lino, F.M.A.; de Sá, L.Z.; Torres, I.M.S.; Rocha, M.L.; Dinis, T.C.P.; Ghedini, P.C.; Somerset, V.S.; Gil, E.S. Voltammetric and spectrometric determination of antioxidant capacity of selected wines. Electrochim. Acta, 2014, 128, 25-31.

[35] Leitão, A.; Andricopulo, A.D.; Oliva, G.; Pupo, M.T.; de Marchi, A.A.; Vieira, P.C.; da Silva, M.F.; Ferreira, V.F.; de Souza, M.C.; Sá, M.M.; Moraes, V.R.; Montanari, C.A. Structure-activity relationships of novel inhibitors of glyceraldehyde-3-phosphate dehydrogenase. Bioorg. Med. Chem. Lett., 2004, 14(9), 2199-2204.

[36] Castro, M.A.; Olea-Azar, C.; Cerecetto, H.; González, M.; Maya, J.D.; Rodríguez-Becerra, J. Potent 5-nitrofuran derivatives inhibitors of Trypanosoma cruzi growth: Electrochemical, spectroscopic and biological studies. Spectrochim. Acta Mol. Biomol. Spectrosc., 2011, 79(2), 312-319.

[37] Aguilera-Venegas, B.; Olea-Azar, C.; Aran, V.J.; Speisky, H. Indazoles: a new top seed structure in the search of efficient drugs against Trypanosoma cruzi. Future Med. Chem., 2013, 5(15), 18431859.

[38] Aguilera-Venegas, B.; Speisky, H. Identification of the transition state for fast reactions: The trapping of hydroxyl and methyl radicals by DMPO-A DFT approach. J. Mol. Graph. Model., 2014, 52, 57-70.

[39] Barriga-Gonzalez, G.; Olea-Azar, C.; Zuñiga-López, M.C.; FolchCano, C.; Aguilera-Venegas, B.; Porcal, W.; González, M.; Cerecetto, H. Spin trapping: an essential tool for the study of diseases caused by oxidative stress. Curr. Top. Med. Chem., 2015, 15, 484495.

[40] Robledo-O'Ryan, N.; Matos, M.J.; Vazquez-Rodriguez, S.; Santana, L.; Uriarte, E.; Moncada-Basualto, M.; Mura, F.; Lapier, M.; Maya, J.D.; Olea-Azar, C. Synthesis, antioxidant and antichagasic properties of a selected series of hydroxy-3-arylcoumarins. Bioorg. Med. Chem., 2017, 25(2), 621-632.

[41] Wolfe, K.; Liu, R. Cellular antioxidant activity (CAA) assay for assessing antioxidants, foods, and dietary supplements. J. Agr. Food Chem., 2007, 55, 8896-8907. 
[42] Mosmann, T. Rapid colorimetric assay for cellular growth and survival: Application to proliferation and cytotoxicity assays. $J$. Immunol. Methods, 1983, 65, 55-63.

[43] Vieites, M.; Otero, L.; Santos, D.; Toloza, J.; Figueroa, R.; Norambuena, E.; Olea-Azar, C.; Aguirre, G.; Cerecetto, H.; Gonzalez, M.; Morello, A.; Maya, J. D.; Garat, B.; Gambino, D.: Platinum(II) metal complexes as potential anti-Trypanosoma cruzi agents. J. Inorg. Biochem., 2008, 102, 1033-1043.
[44] Aldunate, J.; Ferreira, J.; Letelier, M. E.; Repetto, Y.; Morello, A. t-Butyl-4-hydroxyanisole, a novel respiratory chain inhibitor. FEBS Lett., 1986, 195, 295-297.

[45] Okuda, T.; Norioka, M.; Shitara, Y.; Horie, T. Multiple mechanisms underlying troglitazone-induced mitochondrial permeability transition. Toxicol. Appl. Pharmacol., 2010, 248, 242-248.

[46] Silva, T.M.; Peloso, E.F.; Vitor, S.C.; Ribeiro, L.H.; Gadelha, F.R. $\mathrm{O}_{2}$ consumption rates along the growth curve: new insights into Trypanosoma cruzi mitochondrial respiratory chain. J. Bioenerg. Biomembr., 2011, 43, 409-417. 